\title{
Determination Cadmium and Lead Pollution Resources of Ardabil Plain Underground Waters
}

\author{
Saba Hajjabbari, Ebrahim Fataei* \\ Department of Environmental Science, Ardabil Branch, Islamic Azad University, Ardabil, Iran \\ Email: "ebfataei@gmail.com
}

Received 10 June 2016; accepted 18 August 2016; published 22 August 2016

Copyright $(\subseteq 2016$ by authors and Scientific Research Publishing Inc.

This work is licensed under the Creative Commons Attribution International License (CC BY). http://creativecommons.org/licenses/by/4.0/

c) (i) Open Access

\begin{abstract}
Underground water is among the most important sources of drinking water. The aim of this study was to determine the concentrations of heavy metals (cadmium, lead) in providing plain of Ardabil in 2015. This study was cross-sectional, drinking water samples from 100 wells were prepared according to standard procedures. The dimensions of $350 \times 350$ meter grid station via a station in the network were determined by atomic absorption spectrometry AAS analysis of samples. The data obtained were analyzed using SPSS software. The average concentrations of lead, cadmium in groundwater wells in the area were also studied. The mean concentrations measured in the majority of groundwater wells in the plain of Ardabil lower than the allowed amount were designated according to a national standard.
\end{abstract}

\section{Keywords}

Pollution, Groundwater, Drinking, Ardabil Plain

\section{Introduction}

Population growth and rising living standards in many countries resulted in increased demand for various uses of underground water for agriculture, industry, and urban construction. Less pollution of underground water due to the talent as well as a large storage capacity compared to surface water is considered as an important source of water. An increase in electrical conductivity and concentrations of sodium, chloride, sulfate, and nitrate in groundwater is mostly due to human activities such as farming operations, intensive use of fertilizers, water, and industry. Infected surface increases the amount of TDS [1]. Unfortunately, because of the invisibility of many

${ }^{*}$ Corresponding author. 
people about the importance of ground water, the harmful effects of environmental pollution on them are not aware [2]. In the present society, not only water quantity but also water quality attention and research have proven that many diseases are caused by poor quality water. Substances that are potentially harmful to the consumer should be limited and those substances which can affect public acceptance of water should be controlled [3] [4]. Heavy metals including considering the importance of environmental pollutants have attracted attention in scientific communities. Heavy metals in food and tissues of organisms use the food [2]. Heavy metals naturally in groundwater resources due to geology and soils are found in small amounts [3] [4]. Underground water pollution caused by agricultural operations is not a new phenomenon. High consumption of fertilizers in order to achieve maximum agricultural production increased nitrate in groundwater and endangered human health. So with the use of nitrogen fertilizers, a substantial amount of them are underground water. High nitrate concentrations are harm to human health and aquatic animals [5]. According to [3], as the concentration of heavy metals $(\mathrm{Cu}, \mathrm{Cd}, \mathrm{Pb})$ in drinking water in Mashhad had expressed heavy metal compounds through the various imported water sources [6]-[10].

\section{Methodology}

\subsection{The Introduction of the Study Area}

The plain with an area of 95 thousand hectares in the high plateau is between the mountain and into plain packages. Its height is about 1350 meters from all four sides surrounded by mountains, fields and alluvial deposits of eroded mountains surrounding the basin are closed. Plains due to having proper thickness of sediments and soil quality and numerous rivers, the perfect place for farming activities and production of agricultural products, particularly cereals and potatoes is. This plain, with the ground water and in the last half century has long been considered the most important source of water supply for agriculture, industry, and urban and rural drinking. The aquifer until the last two decades, the number of wells and semi-limited and consequently the withdrawal of ground water level were very low. 62 years later and with regard to agricultural development in the region and also and Ardabil urban sprawl and population growth, water extraction from aquifers has been increased for various uses [9] in addition, the network size is 350 in the 350 meters. Also sampled in two seasons of dehydration and over hydration and there is one sample per pixel. Sampling in the summer time months in August and in May 2015 was conducted in the spring. Chemical analysis results in Excel as a database was saved. Position wells were recorded using GPS to map production. Also in this study to analyze the data aggregated statistical software spss test, ANOVA and T-test was used (Figure 1).

\subsection{Results}

Results of the study data obtained from sampling at 100 stations in Ardabil plain dry and wet seasons in 2015 from ground water Table 1 is provided.

The station that was detectable amounts of lead and cadmium in the comparison chart was prepared with the Iranian standards (Diagram 1, Diagram 2).

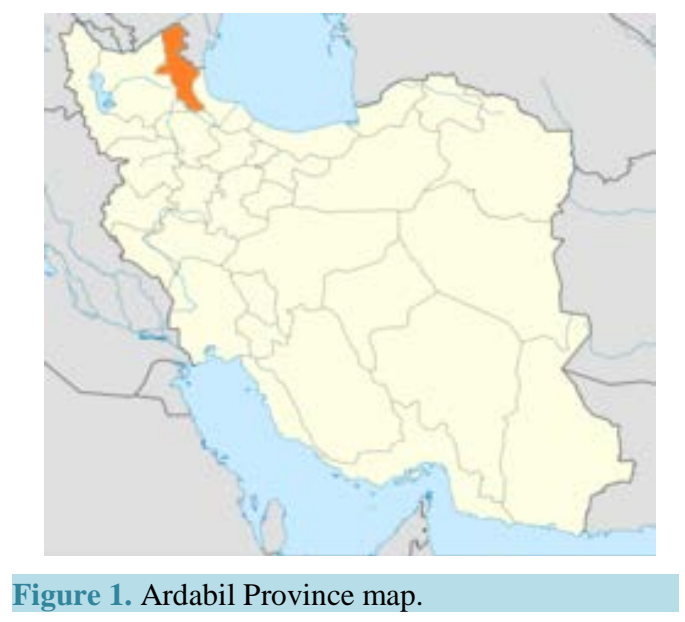


Table 1. Concentration of heavy metals cadmium and lead during dry and wet seasons in the plains of Ardabil.

\begin{tabular}{|c|c|c|c|c|c|c|c|c|c|}
\hline $\begin{array}{l}\text { Station } \\
\text { number }\end{array}$ & $\begin{array}{l}\text { Cadmium } \\
\text { summer }\end{array}$ & $\begin{array}{l}\text { Cadmium } \\
\text { spring }\end{array}$ & $\begin{array}{c}\text { Lead } \\
\text { summer }\end{array}$ & Lead spring & $\begin{array}{c}\text { Station } \\
\text { number }\end{array}$ & $\begin{array}{l}\text { Cadmium } \\
\text { summer }\end{array}$ & $\begin{array}{l}\text { Cadmium } \\
\text { spring }\end{array}$ & $\begin{array}{c}\text { Lead } \\
\text { summer }\end{array}$ & Lead spring \\
\hline 1 & 0.003 & 0.002 & 0.0361 & 0.001 & 51 & 0.000 & 0.000 & 0.0027 & 0.002 \\
\hline 2 & 0.002 & 0.000 & 0.0026 & 0.002 & 52 & 0.000 & 0.000 & 0.0023 & 0.002 \\
\hline 3 & 0.002 & 0.000 & 0.0024 & 0.0018 & 53 & 0.000 & 0.0000 & 0.0017 & 0.001 \\
\hline 4 & 0.003 & 0.001 & 0.003 & 0.0024 & 54 & 0.001 & 0.0007 & 0.0014 & 0.0011 \\
\hline 5 & 0.001 & 0.001 & 0.002 & 0.0014 & 55 & 0.000 & 0.0005 & 0.0019 & 0.0014 \\
\hline 6 & 0.001 & 0.002 & 0.0023 & 0.003 & 56 & 0.000 & 0.000 & 0.0033 & 0.0024 \\
\hline 7 & 0.002 & 0.002 & 0.0022 & 0.002 & 57 & 0.0016 & 0.002 & 0.0026 & 0.002 \\
\hline 8 & 0.0016 & 0.002 & 0.002 & 0.0042 & 58 & 0.002 & 0.000 & 0.0036 & 0.0031 \\
\hline 9 & 0.000 & 0.001 & 0.0017 & 0.000 & 59 & 0.002 & 0.000 & 0.002 & 0.0013 \\
\hline 10 & 0.002 & 0.000 & 0.002 & 0.0018 & 60 & 0.002 & 0.002 & 0.000 & 0.000 \\
\hline 11 & 0.002 & 0.000 & 0.0033 & 0.0029 & 61 & 0.0018 & 0.0013 & 0.0000 & 0.000 \\
\hline 12 & 0.000 & 0.000 & 0.0096 & 0.005 & 62 & 0.001 & 0.000 & 0.0000 & 0.000 \\
\hline 13 & 0.000 & 0.00007 & 0.009 & 0.000 & 63 & 0.001 & 0.0007 & 0.0047 & 0.000 \\
\hline 14 & 0.000 & 0.0000 & 0.0014 & 0.000 & 64 & 0.000 & 0.000 & 0.006 & 0.0033 \\
\hline 15 & 0.0018 & 0.001 & 0.0023 & 0.0015 & 65 & 0.000 & 0.000 & 0.0079 & 0.0023 \\
\hline 16 & 0.0025 & 0.001 & 0.0037 & 0.003 & 66 & 0.000 & 0.001 & 0.005 & 0.0019 \\
\hline 17 & 0.002 & 0.003 & 0.003 & 0.0022 & 67 & 0.000 & 0.000 & 0.0083 & 0.007 \\
\hline 18 & 0.000 & 0.0018 & 0.0012 & 0.001 & 68 & 0.0009 & 0.000 & 0.0089 & 0.007 \\
\hline 19 & 0.000 & 0.002 & 0.001 & 0.0007 & 69 & 0.000 & 0.000 & 0.008 & 0.008 \\
\hline 20 & 0.000 & 0.001 & 0.0012 & 0.000 & 70 & 0.000 & 0.001 & 0.006 & 0.0029 \\
\hline 21 & 0.000 & 0.000 & 0.0015 & 0.000 & 71 & 0.002 & 0.000 & 0.0066 & 0.0024 \\
\hline 22 & 0.000 & 0.000 & 0.0022 & 0.005 & 72 & 0.002 & 0.0016 & 0.002 & 0.0016 \\
\hline 23 & 0.001 & 0.000 & 0.000 & 0.0009 & 73 & 0.003 & 0.001 & 0.0045 & 0.0021 \\
\hline 24 & 0.0014 & 0.000 & 0.0024 & 0.000 & 74 & 0.003 & 0.002 & 0.0032 & 0.002 \\
\hline 25 & 0.001 & 0.002 & 0.003 & 0.001 & 75 & 0.003 & 0.002 & 0.003 & 0.002 \\
\hline 26 & 0.0009 & 0.000 & 0.0007 & 0.000 & 76 & 0.000 & 0.0001 & 0.008 & 0.000 \\
\hline 27 & 0.000 & 0.000 & 0.0007 & 0.000 & 77 & 0.001 & 0.000 & 0.0033 & 0.002 \\
\hline 28 & 0.000 & 0.0017 & 0.0009 & 0.000 & 78 & 0.000 & 0.000 & 0.0078 & 0.0056 \\
\hline 29 & 0.001 & 0.001 & 0.0016 & 0.000 & 79 & 0.000 & 0.000 & 0.0039 & 0.0034 \\
\hline 30 & 0.001 & 0.001 & 0.0016 & 0.000 & 80 & 0.000 & 0.000 & 0.005 & 0.000 \\
\hline 31 & 0.0018 & 0.001 & 0.009 & 0.002 & 81 & 0.0006 & 0.001 & 0.0056 & 0.000 \\
\hline 32 & 0.0016 & 0.000 & 0.016 & 0.01 & 82 & 0.001 & 0.001 & 0.007 & 0.000 \\
\hline 33 & 0.0016 & 0.001 & 0.014 & 0.01 & 83 & 0.001 & 0.000 & 0.009 & 0.0043 \\
\hline 34 & 0.0025 & 0.003 & 0.01 & 0.01 & 84 & 0.003 & 0.002 & 0.01 & 0.0076 \\
\hline 35 & 0.0033 & 0.002 & 0.01 & 0.0088 & 85 & 0.001 & 0.003 & 0.01 & 0.008 \\
\hline
\end{tabular}




\begin{tabular}{ccccccccccc} 
Continued & & & & & & & & & \\
\hline 36 & 0.003 & 0.002 & 0.008 & 0.000 & 86 & 0.004 & 0.003 & 0.0026 & 0.016 \\
37 & 0.002 & 0.003 & 0.016 & 0.01 & 87 & 0.003 & 0.004 & 0.054 & 0.03 \\
38 & 0.0034 & 0.003 & 0.019 & 0.015 & 88 & 0.004 & 0.003 & 0.0467 & 0.033 \\
39 & 0.001 & 0.003 & 0.02 & 0.013 & 89 & 0.002 & 0.004 & 0.015 & 0.01 \\
40 & 0.001 & 0.000 & 0.01 & 0.007 & 90 & 0.001 & 0.002 & 0.013 & 0.009 \\
41 & 0.001 & 0.000 & 0.0094 & 0.005 & 91 & 0.005 & 0.003 & 0.022 & 0.0155 \\
42 & 0.001 & 0.001 & 0.0067 & 0.003 & 92 & 0.005 & 0.002 & 0.0165 & 0.01 \\
43 & 0.001 & 0.000 & 0.0059 & 0.002 & 93 & 0.001 & 0.003 & 0.019 & 0.016 \\
44 & 0.000 & 0.001 & 0.007 & 0.000 & 94 & 0.001 & 0.002 & 0.015 & 0.01 \\
45 & 0.001 & 0.002 & 0.0085 & 0.000 & 95 & 0.003 & 0.002 & 0.0078 & 0.000 \\
46 & 0.000 & 0.0000 & 0.019 & 0.008 & 96 & 0.001 & 0.000 & 0.0056 & 0.000 \\
47 & 0.0008 & 0.001 & 0.0034 & 0.000 & 97 & 0.000 & 0.000 & 0.0014 & 0.0002 \\
48 & 0.001 & 0.001 & 0.0037 & 0.002 & 98 & 0.000 & 0.001 & 0.0012 & 0.0005 \\
49 & 0.002 & 0.003 & 0.0101 & 0.003 & 99 & 0.001 & 0.002 & 0.000 & 0.000 \\
50 & 0.002 & 0.003 & 0.0024 & 0.000 & 100 & 0.000 & 0.000 & 0.005 & 0.000 \\
\hline
\end{tabular}

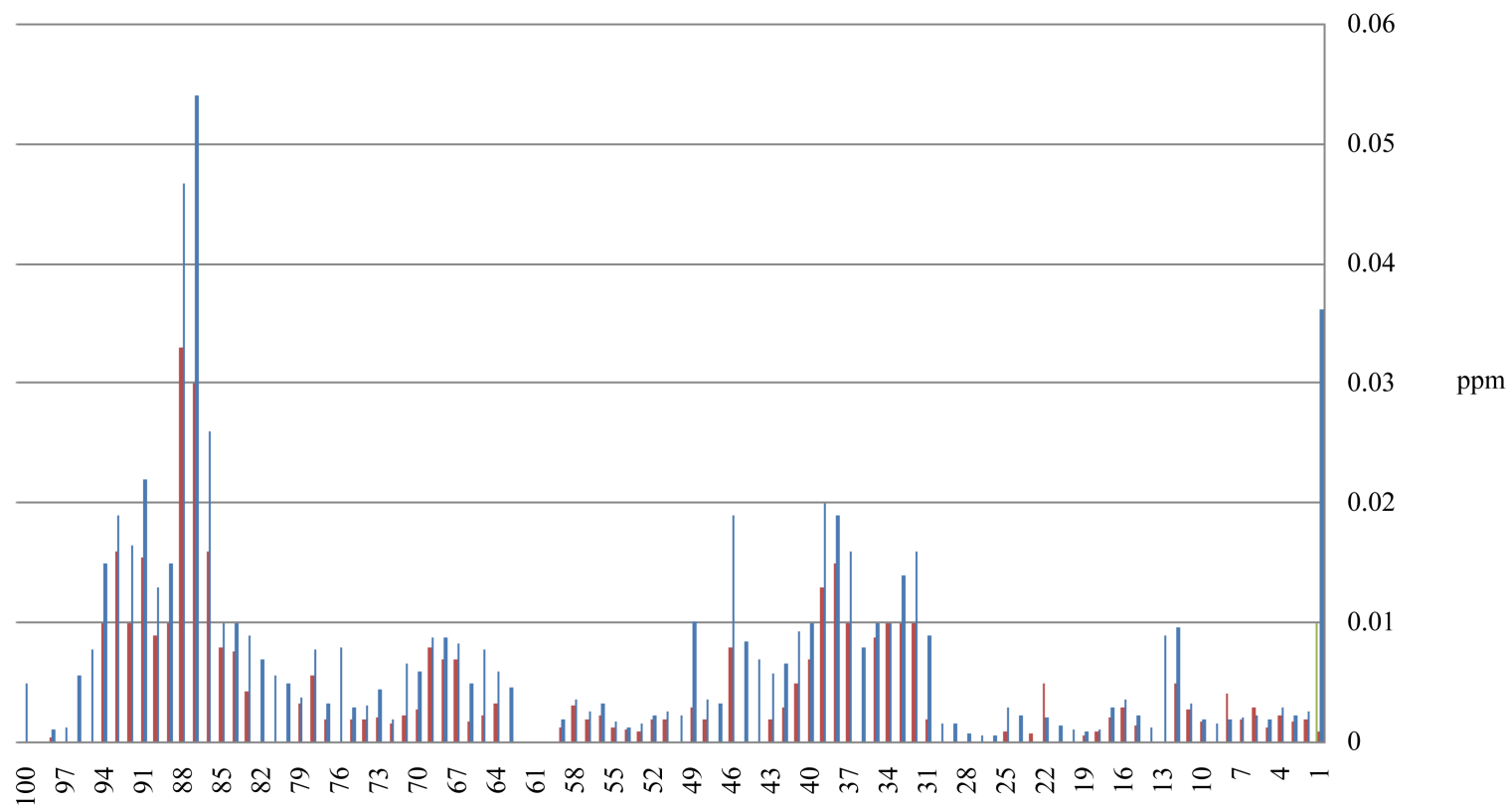

Diagram 1. Comparison of the lead with the Iranian standards.

\subsection{ANOVA Analysis Results}

The results of analysis of variance showed cadmium parameters (Table 2) that evaluated the stations; there was a significant difference at the $1 \%$ level. Results showed that mean comparison test stations (Table 3 ) with an average of 91 ppm 0.004 stations and mobile stations have the highest numbers 1, 17, 36, 38, 49, 50, 74, 75, 84, 95, 35, 34, 37, 89, 39, 86, 87, 88 and 92 groups were polluted stations 12, 14, 21, 22, 27, 46, 51, 52, 53, 56, 64 , $65,67,69,78,79,80$ and 97 with the least amount of pollution have zero value milligrams per liter, respectively.

The results of analysis of variance showed Lead parameters (Table 3) that the stations studied; there was no 


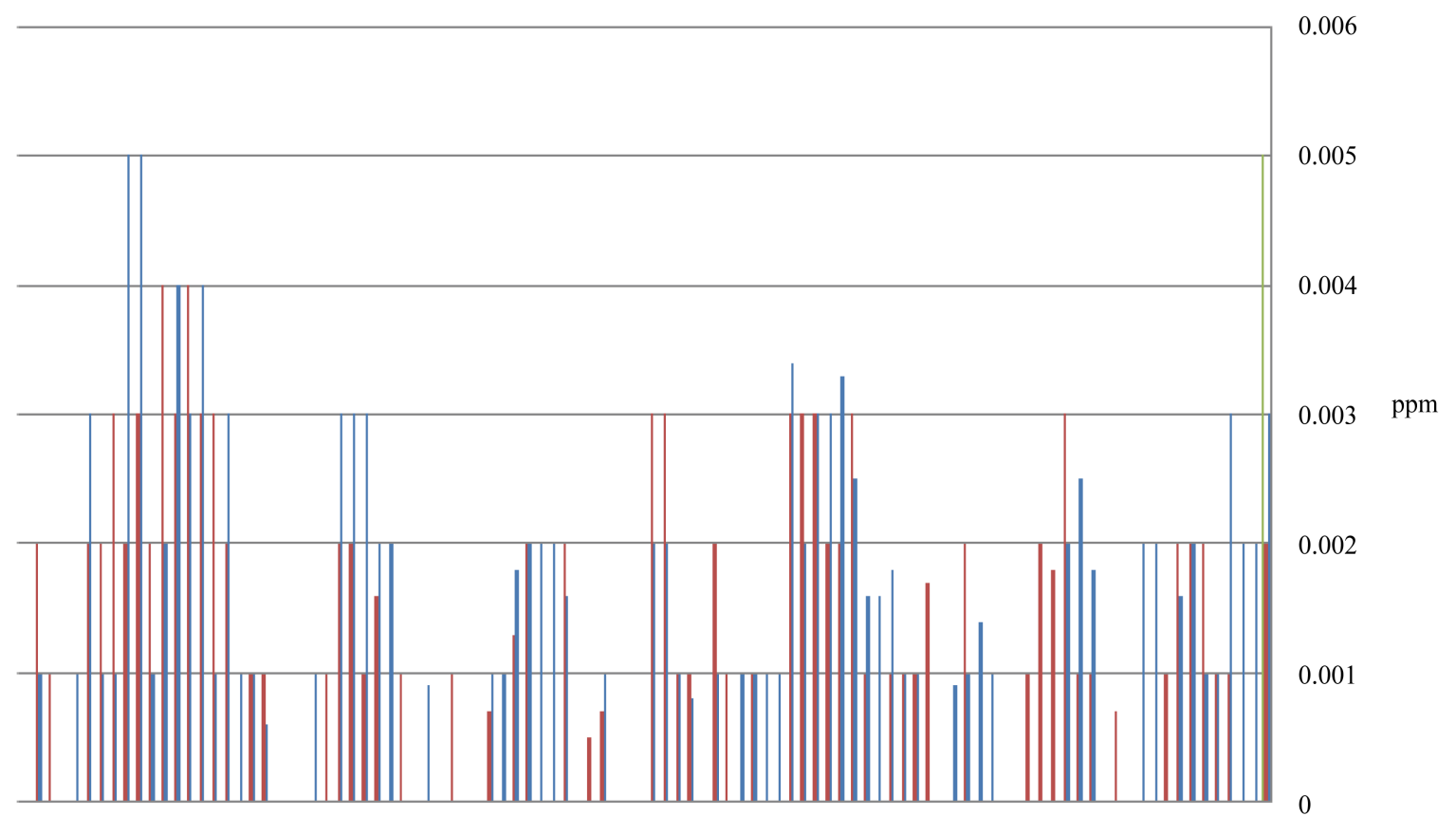

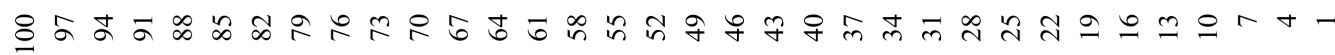

Diagram 2. Comparison of cadmium with the Iranian standards.

Table 2. Analysis of heavy metal cadmium in the plain of Ardabil in 2015.

\begin{tabular}{ccccc}
\hline Sources change & Degrees of freedom & Average of squares & F & Significant level. \\
\hline Station & 99 & $2.141 \mathrm{E}-6$ & 3 & 0.000 \\
Error & 100 & $5.955 \mathrm{E}-7$ & & \\
\hline
\end{tabular}

** Significant at the $1 \%$ level shows.

Table 3. Analysis of heavy metal lead in the plain of Ardabil in 2015.

\begin{tabular}{ccccc}
\hline Sources change & Degrees of freedom & Average of squares & F & Significant level. \\
\hline Station & 99 & $9.813 \mathrm{E}-5$ & 5 & 0.000 \\
Error & 100 & $1.740 \mathrm{E}-5$ & & \\
\hline
\end{tabular}

significant difference in the level of $1 \%$. The comparison showed that the average stations (Table 4) stations 87 and 88, respectively, with an average of 0.042 and $0.03,958 \mathrm{mg}$ had the highest infection rates were higher in statistically class stations No. 26, 27, 60, 61, 62 and 99 with a mean of zero ppm were ranked the lowest.

For comparison Lead and Cadmium parameter values in spring and summer paired samples test were used in this experiment. The results showed that compared pairs of heavy metal lead and cadmium summer statistically significant difference was observed in $1 \%$ probability level (Table 5). Cadmium and cadmium spring summer pairs well with a statistically significant difference in the level of 1 percent.

For measurements with national standard values were used in this experiment One-Sample Test. The results showed that in both heavy metals lead and cadmium in both summer and spring, there was a statistically significant difference at the $1 \%$ level (Table 6).

\section{Conclusions}

Cadmium spring is also based on national standards and EPA (0.005 ppm) lower than standard and cadmium contamination exists. 
Table 4. Comparison of measuring heavy metals lead and cadmium of Ardabil plain Duncan (ppm).

\begin{tabular}{|c|c|c|c|c|c|c|c|c|c|}
\hline \multirow{3}{*}{$\begin{array}{c}\begin{array}{c}\text { Station } \\
\text { number }\end{array} \\
1\end{array}$} & \multicolumn{4}{|c|}{ Heavy metals } & \multirow{3}{*}{$\begin{array}{c}\begin{array}{c}\text { Station } \\
\text { number }\end{array} \\
51\end{array}$} & \multicolumn{4}{|c|}{ Heavy metals } \\
\hline & \multicolumn{2}{|c|}{ Lead } & \multicolumn{2}{|c|}{ Cadmium } & & \multicolumn{2}{|c|}{ Lead } & \multicolumn{2}{|c|}{ Cadmium } \\
\hline & 0.0025 & e-a & 0.01855 & bcd & & 0.0000 & g & 0.0023 & klmn \\
\hline 2 & 0.001 & gfed & 0.0023 & klmn & 52 & 0.0000 & g & 0.00215 & klmn \\
\hline 3 & 0.001 & gfed & 0.0021 & klmn & 53 & 0.0000 & g & 0.00135 & $\mathrm{mn}$ \\
\hline 4 & 0.002 & f-b & 0.0027 & $j-n$ & 54 & 0.00085 & defg & 0.00125 & $\mathrm{mn}$ \\
\hline 5 & 0.001 & gfed & 0.0017 & $\operatorname{lmn}$ & 55 & 0.00025 & fg & 0.00165 & $\operatorname{lmn}$ \\
\hline 6 & 0.0015 & g-c & 0.00265 & j-n & 56 & 0.0000 & g & 0.00285 & i-n \\
\hline 7 & 0.002 & f-b & 0.0021 & klmn & 57 & 0.0018 & b-g & 0.0023 & klmn \\
\hline 8 & 0.0018 & b-g & 0.0031 & h-n & 58 & 0.001 & defg & 0.00335 & h-n \\
\hline 9 & 0.0005 & fg & 0.00085 & $\mathrm{mn}$ & 59 & 0.001 & defg & 0.00165 & $\operatorname{lmn}$ \\
\hline 10 & 0.001 & defg & 0.0019 & $\operatorname{lmn}$ & 60 & 0.002 & b-f & 0.0000 & $\mathrm{n}$ \\
\hline 11 & 0.001 & defg & 0.0031 & h-n & 61 & 0.0015 & c-g & 0.0000 & $\mathrm{n}$ \\
\hline 12 & 0.0000 & g & 0.0073 & e-n & 62 & 0.0005 & fg & 0.0000 & $\mathrm{n}$ \\
\hline 13 & 0.00035 & fg & 0.0045 & h-n & 63 & 0.00085 & defg & 0.00235 & klmn \\
\hline 14 & 0.0000 & g & 0.0007 & $\mathrm{mn}$ & 64 & 0.0000 & g & 0.00465 & h-n \\
\hline 15 & 0.0014 & c-g & 0.0019 & $\operatorname{lmn}$ & 65 & 0.0000 & g & 0.0051 & h-n \\
\hline 16 & 0.00175 & b-g & 0.00335 & h-n & 66 & 0.0005 & fg & 0.00345 & h-n \\
\hline 17 & 0.0025 & a-e & 0.0026 & j-n & 67 & 0.0000 & g & 0.00765 & e-n \\
\hline 18 & 0.0009 & defg & 0.0011 & $\mathrm{mn}$ & 68 & 0.00045 & fg & 0.00795 & e-n \\
\hline 19 & 0.001 & defg & 0.00085 & $\mathrm{mn}$ & 69 & 0.0000 & g & 0.0084 & d-n \\
\hline 20 & 0.0005 & fg & 0.0006 & $\mathrm{mn}$ & 70 & 0.00005 & fg & 0.00445 & h-n \\
\hline 21 & 0.0000 & g & 0.00075 & $\mathrm{mn}$ & 71 & 0.001 & defg & 0.0045 & h-n \\
\hline 22 & 0.0000 & g & 0.0036 & h-n & 72 & 0.0018 & b-g & 0.0018 & $\operatorname{lmn}$ \\
\hline 23 & 0.0005 & fg & 0.00045 & $\mathrm{mn}$ & 73 & 0.002 & b-f & 0.0033 & h-n \\
\hline 24 & 0.0007 & efg & 0.0012 & $\mathrm{mn}$ & 74 & 0.0025 & a-e & 0.0026 & $j-n$ \\
\hline 25 & 0.0015 & c-g & 0.002 & klmn & 75 & 0.0025 & a-e & 0.0025 & j-n \\
\hline 26 & 0.00045 & fg & 0.00035 & $\mathrm{n}$ & 76 & 0.0005 & fg & 0.004 & h-n \\
\hline 27 & 0.0000 & g & 0.00035 & $\mathrm{n}$ & 77 & 0.0005 & fg & 0.00265 & $\mathrm{j}-\mathrm{n}$ \\
\hline 28 & 0.00085 & defg & 0.00045 & $\mathrm{mn}$ & 78 & 0.0000 & g & 0.0067 & g-n \\
\hline 29 & 0.001 & defg & 0.0008 & $\mathrm{mn}$ & 79 & 0.0000 & g & 0.00365 & h-n \\
\hline 30 & 0.001 & defg & 0.0008 & $\mathrm{mn}$ & 80 & 0.0000 & g & 0.0025 & j-n \\
\hline 31 & 0.0014 & c-g & 0.0055 & h-n & 81 & 0.0008 & defg & 0.0028 & i-n \\
\hline 32 & 0.0008 & defg & 0.013 & b-j & 82 & 0.001 & defg & 0.0035 & h-n \\
\hline 33 & 0.0013 & c-g & 0.012 & b-l & 83 & 0.0005 & fg & 0.00665 & g-n \\
\hline 34 & 0.00275 & abcd & 0.01 & c-n & 84 & 0.0025 & a-e & 0.0088 & c-n \\
\hline
\end{tabular}




\section{Continued}

\begin{tabular}{ccccccccccccc}
\hline 35 & 0.00265 & a-e & 0.0094 & c-n & 85 & 0.002 & b-f & 0.009 & c-n \\
36 & 0.0025 & a-e & 0.004 & h-n & 86 & 0.0035 & ab & 0.021 & b \\
37 & 0.003 & abc & 0.013 & b-j & 87 & 0.0035 & ab & 0.042 & a \\
38 & 0.0025 & a-e & 0.017 & b-f & 88 & 0.0035 & ab & 0.03985 & a \\
39 & 0.0032 & abc & 0.0165 & b-g & 89 & 0.003 & abc & 0.0125 & b-k \\
40 & 0.0005 & fg & 0.0085 & d-n & 90 & 0.0015 & c-g & 0.011 & b-m \\
41 & 0.0005 & fg & 0.0072 & f-n & 91 & 0.004 & a & 0.01875 & bc \\
42 & 0.001 & defg & 0.00485 & h-n & 92 & 0.0035 & ab & 0.01325 & b-i \\
43 & 0.0005 & fg & 0.00395 & h-n & 93 & 0.002 & b-f & 0.0175 & bcde \\
44 & 0.0005 & fg & 0.0035 & h-n & 94 & 0.0015 & c-g & 0.0125 & b-k \\
45 & 0.0015 & c-g & 0.00425 & h-n & 95 & 0.0025 & a-e & 0.0039 & h-n \\
46 & 0.0000 & g & 0.0135 & b-h & 96 & 0.0005 & fg & 0.0028 & i-n \\
47 & 0.0009 & defg & 0.0017 & lmn & 97 & 0.0000 & g & 0.0008 & mn \\
48 & 0.001 & defg & 0.00285 & i-n & 98 & 0.0005 & fg & 0.00085 & mn \\
49 & 0.0025 & a-e & 0.00655 & g-n & 99 & 0.0015 & c-g & 0.0000 & g & n & 0.0025 & j-n
\end{tabular}

Table 5. Test paired samples test for heavy metals lead and cadmium contamination of ground water in the spring and summer in Ardabil plain.

\begin{tabular}{|c|c|c|c|c|c|c|c|c|c|}
\hline & \multirow{2}{*}{ C. } & \multirow{2}{*}{ Average } & \multirow{2}{*}{$\begin{array}{c}\text { The standard } \\
\text { deviation }\end{array}$} & \multirow{2}{*}{$\begin{array}{c}\text { The average } \\
\text { standard } \\
\text { deviation }\end{array}$} & \multicolumn{2}{|c|}{$\begin{array}{l}\text { 95\% Confidence interval of } \\
\text { the difference }\end{array}$} & \multirow[t]{2}{*}{$\mathrm{t}$} & \multirow{2}{*}{ Df } & \multirow{2}{*}{$\begin{array}{l}\text { Sig. } \\
\text { (2-tailed) }\end{array}$} \\
\hline & & & & & Lower & Upper & & & \\
\hline 1 Pair & $\begin{array}{l}\text { Lead and cadmium } \\
\text { summer }\end{array}$ & -0.0043915 & 0.0071065 & 0.0005025 & -0.0053824 & -0.0034006 & -8 & 199 & 0.000 \\
\hline Pair 2 & $\begin{array}{c}\text { Cadmium and cadmium } \\
\text { spring summer }\end{array}$ & 0.0001810 & 0.0010817 & 0.0001082 & -0.0000336 & 0.0003956 & 1 & 99 & 0.097 \\
\hline Pair 2 & $\begin{array}{l}\text { Lead and lead spring } \\
\text { summer }\end{array}$ & 0.0033740 & 0.0048634 & 0.0004863 & 0.0024090 & 0.0043390 & 6 & 99 & 0.000 \\
\hline
\end{tabular}

Table 6. Paired samples test for heavy metals lead and cadmium contamination of ground water in the spring and summer in Ardabil plain.

\begin{tabular}{|c|c|c|c|c|c|c|}
\hline & \multirow{2}{*}{$\mathrm{T}$} & \multirow{2}{*}{$\mathrm{df}$} & \multirow{2}{*}{ Sig. (2-tailed) } & \multirow{2}{*}{ Mean } & \multicolumn{2}{|c|}{$95 \%$ Confidence interval of the difference } \\
\hline & & & & & Lower & Upper \\
\hline & & & & Test Value $=0.005$ & & \\
\hline Cadmium summer & -45 & 100 & 0.000 & -0.0037895 & -0.003952 & -0.003627 \\
\hline \multirow[t]{2}{*}{ Cadmium spring } & -34 & 99 & 0.000 & -0.0038800 & -0.004102 & -0.003658 \\
\hline & & & & Test Value $=0.01$ & & \\
\hline Lead summer & -8 & 199 & 0.000 & -0.0043980 & -0.005456 & -0.003340 \\
\hline Lead spring & -10 & 99 & 0.000 & -0.0060850 & -0.007211 & -0.004959 \\
\hline
\end{tabular}


According to WHO, (ppm 0.003) summer cadmium in five sampling stations including (35-39-86-91-92) and cadmium spring at two stations (87-89) are higher than the standard. Also standard EPA (ppm 0.015) indicates lead summer in 12 stations (1-32-37-38-39-46-86-87-88-91-92-93) and lead spring in five sampling stations (86-87-88-91-93) are higher than the standard. Emissions in the vicinity are in Mollayousef, Guradel-Araluy Bozorg, Kalkhoran, Gorgan, Fuladlu, Dalilghureh, Jobbedar, Gharjur, Mireni, Khiyarak, GazSamian, Dowlatabad, Ali Bulaghi villages.

\section{References}

[1] Alidadi, H. (2011) The Concentration of Heavy Metals, Chromium, Cadmium, Lead in Drinking Water in Mashhad. Medical Journal, No. 116, 1-12.

[2] Davis, A., Kempton, J.H. and Nicholson, A. (1994) Ground Water Transport of Arsenic and Chromium at a Historical Tannery. Applied Geochemistry, 9, 569-582.

[3] Eghbali Shamsabadi, P. (2010) The Heavy Metals Chromium, Cadmium, Lead and Organic Materials in Their Structural Sefidrood Attitude on the Origin of the Earth. Journal of Islamic Azad University of Ahvaz Specialized Wetland, No. 3, 20-45.

[4] Davis, A. (1995) Ground Water Transport. Applied Geochemistry, 10, 150-165.

[5] Minareji, E. (2009) The Amount of Heavy Metals Cadmium, Chromium, Copper, Iron, Nickel, Lead in Water, Sediments and Fish Tissue Avshar Carp Lake. Iran.

[6] Asadi, A. (2003) The State of Sanitation in 22 Tehran and Its Effects on the Development Plan. A Graduate Thesis Environmental Health Engineering, School of Public Health, Tehran University of Medical Sciences, Tehran.

[7] Rezvani, M. (2013) Assessment of Contamination Levels of Heavy Metals Cadmium, Cobalt, Lead, Zinc, Manganese Bkhvn Eshtehard. Science and Environmental Engineering, No. 1, 45-53.

[8] Shirani, Z. (2013) The Assessment of Groundwater Pollution in the Urban Environment. Human and Environmental Quarterly, No. 24, 85.

[9] (2015) Groundwater Challenges and Solutions. Public Relations Logic of Ardabil Water.

[10] Hamzeh, M.A. (2009) To Evaluate Changes in the Concentration of Lead, Zinc, Copper and Cadmium In-City Environment, Science and Technology, the Environment. Journal of Science and Technology, 2, 20-25.

\section{Submit or recommend next manuscript to SCIRP and we will provide best service for you:}

Accepting pre-submission inquiries through Email, Facebook, LinkedIn, Twitter, etc. A wide selection of journals (inclusive of 9 subjects, more than 200 journals)

Providing 24-hour high-quality service

User-friendly online submission system

Fair and swift peer-review system

Efficient typesetting and proofreading procedure

Display of the result of downloads and visits, as well as the number of cited articles

Maximum dissemination of your research work

Submit your manuscript at: http://papersubmission.scirp.org/ 